\title{
BUILDINGS IN HIGH RESOLUTION SAR IMAGES - IDENTIFICATION BASED ON CITYGML DATA
}

\author{
S. Auer ${ }^{\mathrm{a}, *}$, A. Donaubauer ${ }^{\mathrm{b}}$ \\ ${ }^{a}$ Remote Sensing Technology Institute, German Aerospace Center (DLR), 82234 Oberpfaffenhofen, Germany - stefan.auer@dlr.de \\ ${ }^{\mathrm{b}}$ Chair of Geoinformatics, Technische Universität München, 80333 München, Germany - andreas.donaubauer@tum.de
}

\author{
Commission VI, WG VI/4
}

KEY WORDS: High resolution SAR, SAR simulation, GIS, CityGML, Interpretation, TerraSAR-X, Urban Areas

\begin{abstract}
:
Motivated by the distinct appearance of facades in high resolution SAR images with respect to signal incidence angles and polarizations, this paper introduces a way to fuse SAR imagery and 3D GIS (geoinformation system) data (format: CityGML) based on SAR simulation methods. To this end, the known building geometry is used to simulate the extent of building layover for identifying the related image parts in high resolution TerraSAR-X images. The simulated SAR images are generated and geocoded by an automated processing chain which is initialized by the automated parsing of the CityGML dataset and the TerraSAR-X orbit file. Confirming the functionality of the developed interface between simulation and CityGML, first results are presented for an urban scene in the Munich city center in order to discuss future opportunities in the context of change detection applications.
\end{abstract}

\section{INTRODUCTION}

High resolution SAR images are of special interest if rapid response is necessary in urgent situations such as flooding or earth quakes. In this context, the reason is not only related to the availability of imagery due to the independence on weather or daytime. What is more, SAR images contain important information which is complementary to other sensor data. As an example, the imaging concept of SAR favors the salient appearance of facade structures in so-called layover areas which are hardly visible to optical satellite sensors.

For identifying the status or change of urban objects, the main difficulty is related to the identification of layover areas corresponding to individual buildings or even individual facades. A look to the literature reveals that possible concepts may be based on the integration of known object geometry from LiDAR data (Tao et al., 2014) but also on the analysis of interferometric SAR data (Thiele et al., 2013) or SAR image amplitudes (Ferro et al., 2013). In that regard, the identification of layover parts is either limited by the included scene model (density of LiDAR point cloud or spacing of DSM) or the layover property in the SAR data (prominent appearance of signatures, speckle, and coherence). However, the extraction of building layover offers new opportunities as an object-based analysis can be pursued for equal or changing imaging perspectives. Besides basic measures of building layover (e.g. areal features or intensity distributions), methods for characterizing linear signatures (Simonetto et al., 2005, Wegner et al., 2010) or point-like signatures may be applied in this context (Soergel et al., 2006, Michaelsen et al., 2006, Auer et al., 2015).

This paper addresses two main aspects related to the distinct analysis of buildings in high resolution SAR images. First, the typical appearance of facade layover in high resolution SAR images is exemplified and discussed with respect to different signal incidence angles and polarizations (Section II), giving an impression on information contained in the related image signatures. In this context, the extraction of building layover in SAR images is motivated. Serving this need, a simulation method based on (Tao

\footnotetext{
*Corresponding author.
}

et al., 2014) is reported in the second part of the paper. Building layover parts are identified in the SAR image based on the integration of prior knowledge given by a CityGML dataset. To the knowledge of the authors, the development of an interface between CityGML and SAR data in a framework of absolute coordinates is presented for the first time (Section III). Simulation results of a case study are shown in Section IV followed by a discussion in Section $\mathrm{V}$ in order to indicate the potential of the CityGML data in the context of change detection. Section VI concludes the paper and provides an outlook to future work.

\section{FACADE LAYOVER - PROPERTIES AND POTENTIALS}

The radar signal response of facades mainly depends on the surface material of imaged objects, the signal wavelength and polarization as well as the imaging angles (signal incidence angle, facade rotation). In case of high resolution SAR sensors, facades are represented by high numbers of pixels. Then, the radiometric and geometric distribution of prominent layover signatures can be used to extract knowledge about the facade status or structure. Two examples for prominent facade signatures are given below for the city center of Berlin (Fig. 1 and Fig. 4). The layover areas, marked with red frames in the figures, have been manually selected with the constraint of equal layover sizes for equal signal incidence angles to support the comparability. The SAR images have been captured by TerraSAR-X in high resolution spotlight mode with two incidence angles $\left(30^{\circ} / 51^{\circ}\right)$ and two polarizations $(\mathrm{HH} / \mathrm{VV})$. The time span between the corresponding data takes of $\mathrm{HH}$ and $\mathrm{VV}$ is one revisit period (11 days) in order to minimize the impact of man-made changes. In particular, the focus is on the influence of the building type (residential building vs. skyscraper), the signal polarization (HH vs. VV), the building orientation (line-of-flight vs. rotated), and a variation of the signal incidence angle. As a first hint towards the exploitation of layover pixels, the layover areas are analyzed for their intensity distributions and the number of point-like signatures. In that regard, we consider two findings reported in the literature: A.) the expected dominance of $\mathrm{HH}$ over $\mathrm{VV}$ in the context of point targets (Gernhardt and Bamler, 2012) and B.) the log-normal dis- 


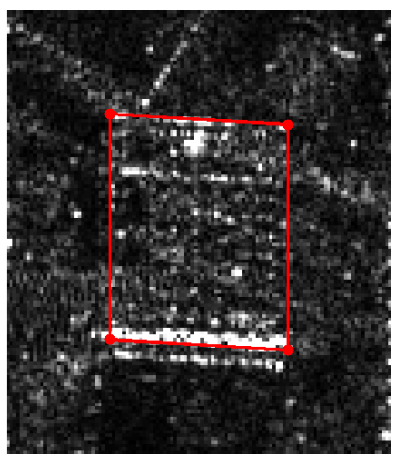

(a) $30^{\circ}, \mathrm{HH}$

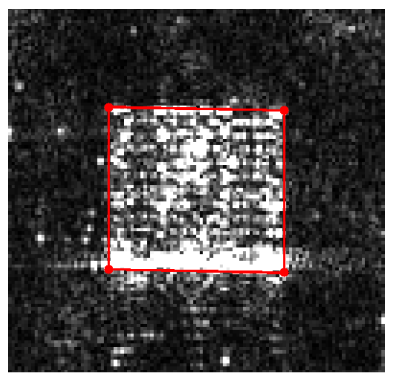

(c) $51^{\circ}, \mathrm{HH}$

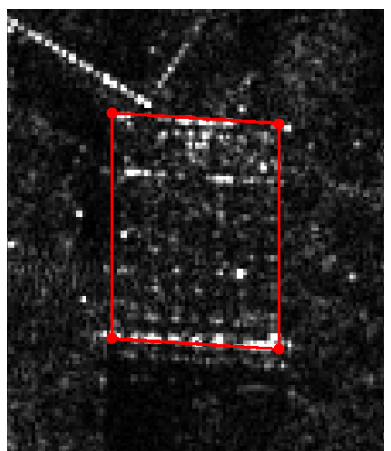

(b) $30^{\circ}, \mathrm{VV}$

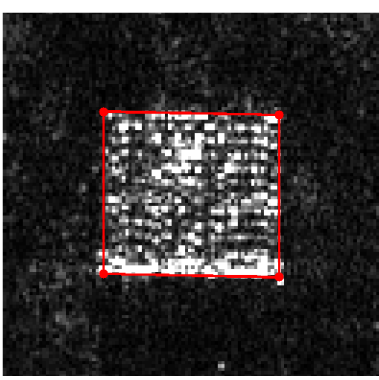

(d) $51^{\circ}, \mathrm{VV}$
Figure 1. Facade at Hansaviertel, Berlin. Extracted facade layover areas indicated by red frames. HH: signal emitted and detected in horizontal plane. VV: signal emitted and detected in vertical plane. Time difference between $\mathrm{HH}$ and VV data takes: 11 days.

tribution as an approximate function for urban areas (Oliver and Quegan, 2004). For each building, the intensities are clipped at the same level for $\mathrm{HH}$ and VV and visualized in 8-bit gray values. The analysis of the distributions is conducted with the full range of intensities.

The first example is related to a facade of a residential building, located in the Hansaviertel, which is covered with columns of windows at the outer ends and columns of balconies in the center. Despite the balconies, the corner reflectors (windows, doors) are located on a plane and mostly part of a uniform grid. The facade is only little rotated with respect to the line-of-flight of TerraSAR-X. Fig. 1 shows the corresponding layover areas for two incidence angles and two polarizations. Visually, the regularity of the facade structure is not present for the incidence angle of $30^{\circ}$ and barely distinguishable for $51^{\circ}$. Intensities in $\mathrm{HH}$ seem to dominate those in VV. In particular, the corner line at the bottom end of the layover is more prominent in $\mathrm{HH}$. The visual impression is confirmed by the intensity distribution (Fig. 2) and characteristic numbers (see columns 1-3 in Table 1) where the mean and median intensity is higher in HH. The standard deviation is higher in $\mathrm{HH}$ as well, meeting the visual perception of speckle in the images. Considering all layover pixels, the assumption of log-likelihood distributions fits well to the data with except of the histogram peaks (Fig. 2). Considering only local maxima of strong intensity, which are presumably related to corner reflections, the assumption only holds as a rough approximation (Fig. 3). Accordingly, the distributions may be used for identifying strong outliers in intensity, e.g., dominating but isolated point targets outside a grid of uniform corner reflectors. Comparable to the selection of all layover pixels, the mean intensities, median intensities and standard deviations of the local maxima are higher in HH (see columns 3-6 in Table 1). Counting the number of prominent local maxima - with intensities higher than the mean

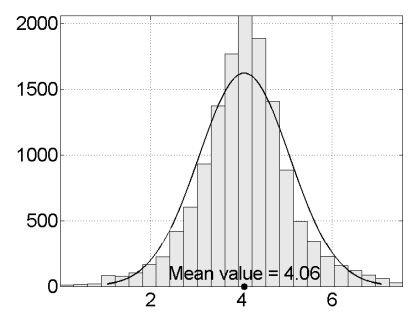

(a) Layover, $30^{\circ}, \mathrm{HH}$

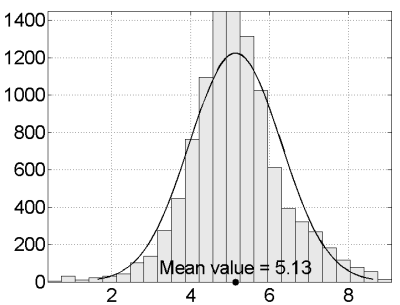

(c) Layover, $51^{\circ}, \mathrm{HH}$ (b) Layover, $30^{\circ}, \mathrm{VV}$

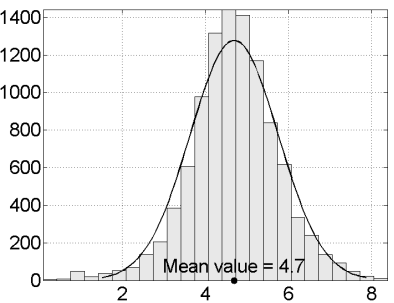

(d) Layover, $51^{\circ}, \mathrm{VV}$

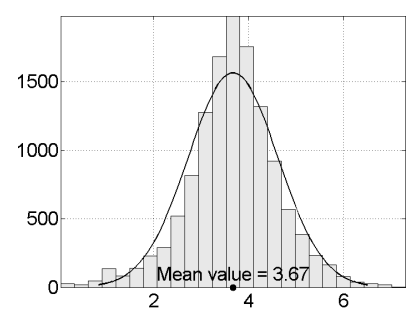

Figure 2. Hansaviertel, intensity distribution of layover pixels for different polarizations and incidence angles.

layover intensity - reveals no trend (see last column in Table 1).

The second example relates to a skyscraper at the Alexanderplatz which is rotated with respect to the line-of-flight of TerraSAR-X. Covered by a combination of regularly arranged metallic structures and glass, the four data takes (combination of two incidence angles and two polarizations) reveal different appearances of the facade layover area (see Fig. 4). For the incidence angle of $51^{\circ}$, the visual perception of the $\mathrm{HH}$ and $\mathrm{VV}$ images is similar. In contrast, a signature pattern is distinguishable in the $\mathrm{HH}$ image for $30^{\circ}$ (linear arrangement of point signatures for each floor) which is not present in the VV image. The intensities of corner lines at the bottom ends of the layovers to be comparable.

As shown in Fig. 5, the intensity distribution of layover pixels can be described by a log-normal distribution for $30^{\circ}$. The layover pixels pertinent to the incidence angle of $51^{\circ}$, however, only roughly follow this assumption. Comparable to the Hansaviertel building, the mean and median intensities are higher in $\mathrm{HH}$ (see columns 1-3 in Table 1). The standard deviations are comparable for both angles. Considering only local maxima in the layover, the log-normal distribution can be used as a rough approximation (Fig. 6). Again, intensities in $\mathrm{HH}$ are dominant compared to VV (mean and median intensity), whereas the standard deviations are comparable (see columns 4-6 in Table 1). As for the residential building above, the comparison of $\mathrm{HH}$ and $\mathrm{VV}$ indicates no trend on the number of dominant point targets which represent structural elements at the skyscraper facade (last column in Table 1).

Considering also results of other facades not reported in the paper, the following conclusions can be drawn at this point:

- The facade examples reveal that the intensity level of $\mathrm{HH}$ is higher than VV for the same incidence angle. From a theoretical viewpoint, the reason may be related to non-metallic surfaces participating at the signal reflection process. Then, specular reflections near the Brewster angle lead to low Fresnel reflection factors in vertical polarization. The effect is almost negligible for metallic surfaces as the Brewster angle is then close to $90^{\circ}$ with little signal loss). Horizontally polarized signals are not affected by this effect of signal loss as the signal reflectivity increases steadily with the reflection angle, i.e. no Brewster angle occurs. The result on a 


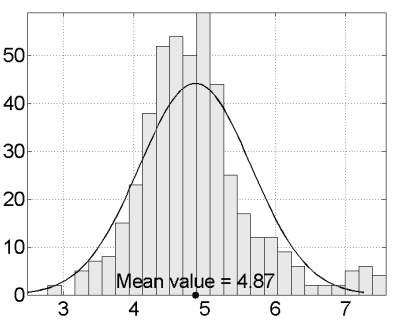

(a) Maxima, $30^{\circ}, \mathrm{HH}$

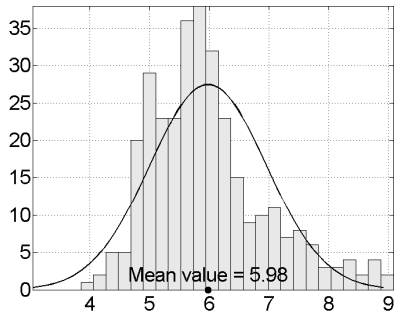

(c) Maxima, $51^{\circ}, \mathrm{HH}$

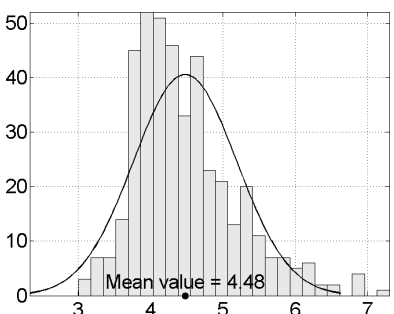

(b) Maxima, $30^{\circ}, \mathrm{VV}$

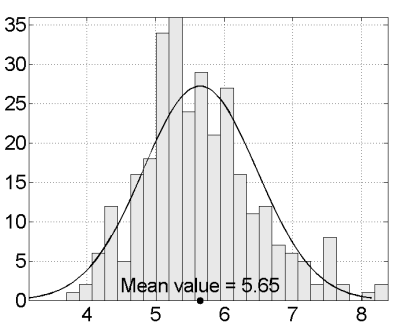

(d) Maxima, $51^{\circ}, \mathrm{VV}$
Figure 3. Hansaviertel, intensity distribution of maxima for different polarizations and incidence angles.

trihedral (corner reflector) can be shown by a simple simulation experiment where two surfaces are made of metal with little signal loss and one surface of glass containing a silver compound for light protection (comparable to a window on a building facade). In this context, the reflection components of the glass and silver compound (located at the interior glass surface) are summed to derive the final signal strength. For the sake of simplicity, signal refraction and attenuation in the glass surface are neglected. The amount of light protection is steered by a weight factor, representing the percentage of glass surface covered by silver. For all signals leaving the corner reflector, the reflection factors for $\mathrm{HH}$ are always higher than for VV (see different cases summarized in Table 2), independently of the amount of light protection.

- The intensity variation in $\mathrm{HH}$ tends to be stronger than or equal to VV. As expected, the intensity level of the layover increases with the incidence angle as more signal contributions are condensed in one range resolution cell.

- The log-normal distribution is applicable as a rough representation of layover pixels. This extends the assumption of (Oliver and Quegan, 2004) where the log-normal distribution is generally related to urban clutter. The analysis in this paper indicates that this assumption is applicable to individual facades as well with dominant signatures related to structural details. Considering local maxima only, the assumption can be used to identify strong outliers in intensity. However, the applicability of the log-normal distribution is moderate for strongly rotated facades and facades with a low number of local maxima (low number of entries in the histogram).

- No general trend has been identified in the number of dominant pixels in building layover (HH vs. VV). Hence, the higher number of salient point targets in HH stacks (see (Gernhardt and Bamler, 2012)) is not confirmed for single SAR images.

- The appearance of facade regularities in the SAR image not only depends on the geometry (object structure and rotation; imaging perspective) but also on the signal polarization (see example related to $30^{\circ}$ in Fig. 4). Therefore, the analysis of layover areas should be based on general measures (e.g. considering the intensity distribution) which can be optionally extended by feature-based concepts as, e.g., shown in (Auer et al., 2015).

As shown above, the appearance of facades in SAR images can be manifold. Nonetheless, distinct layover properties can be analyzed for individual buildings or facades, given the layover extent. To this end, however, the facade layover has to be identified and extracted beforehand in the SAR image. Following this objective, the remainder of this paper extends a simulation method reported in (Tao et al., 2014) by implementing an interface to the GIS standard CityGML as an external data source. The building geometry defined in the CityGML dataset is used as prior knowledge to directly simulate the extent of building layover in the SAR image plane.

\section{SAR SIMULATION FOR BUILDING IDENTIFICATION}

\subsection{Processing Chain for SAR Simulation}

CityGML data and TerraSAR-X data are fused in the framework of a SAR simulator named RaySAR (Auer, 2011). Extended to an automated processing chain for simulating geocoded data named GeoRaySAR, the resulting images can be directly superposed to the TerraSAR-X data in order to identify buildings of interest (Tao et al., 2014). So far, the processing chain has been adapted to import geometric information from LiDAR data and digital surface models (DSMs). In this paper, first steps are reported to open the processing chain for GIS data.

The core of the simulator is based on an extended version of the open-source ray tracer POV-Ray. Concentrating on the geometric part (while using simplified radiometric parameters), artificial signals (rays) are followed a virtual urban scene in order to detect signal reflections of different reflection levels. In that regard, reflection levels 1-3 are of special interest for 2.5D object models, whereas higher reflection levels are of use for analyzing the impact of detailed facade structures. The geometric component is based on a scene model in absolute coordinates and the orbit parameters defining the point-of-view with respect to the scene (incidence angle, rotation angle of buildings). To this end, the scene geometry is parsed into a language interpretable by the SAR simulator (POV-Ray model format; processed in C++ code). Details on the potentials and limitations of the SAR simulation method can be found in (Auer, 2011).

\subsection{CityGML-Data as Data Source}

CityGML (City Geography Markup Language) is an international data standard for geoinformation systems (GIS) which has been defined by the Open Geospatial Consortium (OGC) (Groeger et al., 2012). CityGML defines an object-oriented, application neutral information model for 3D city and landscape models. Using CityGML, real world entities such as buildings, roads and water bodies are modeled with respect to their geometric and semantic properties as well as with their appearance in five discrete levels of detail (LOD). In addition to the information model which is described by a UML class diagram, the CityGML standard defines an XML-based data encoding.

CityGML has been selected for the study at hand for two reasons: first, CityGML is considered to be widely adopted (e.g. the 


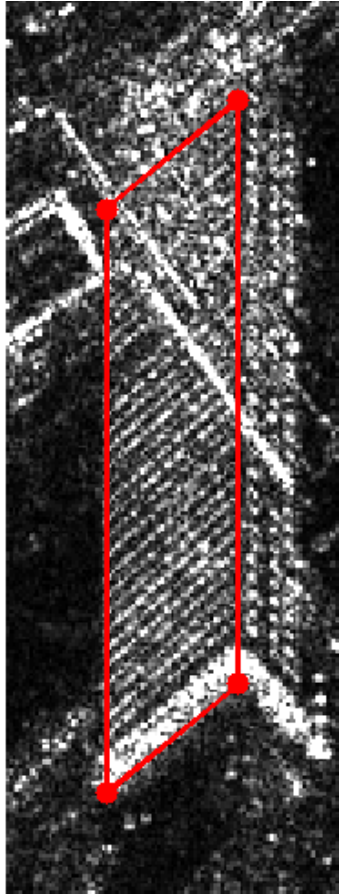

(a) $30^{\circ}, \mathrm{HH}$

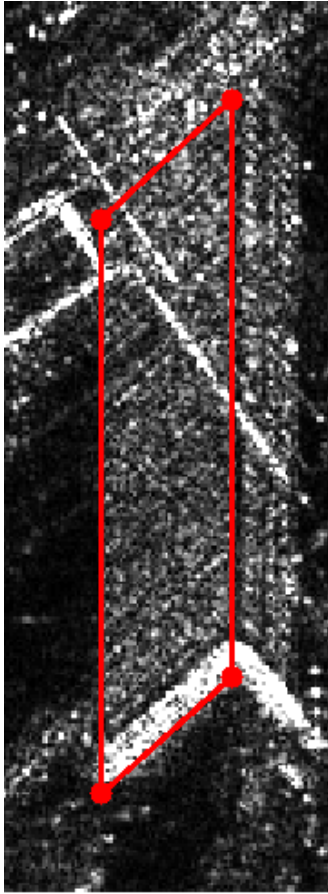

(b) $30^{\circ}, \mathrm{VV}$

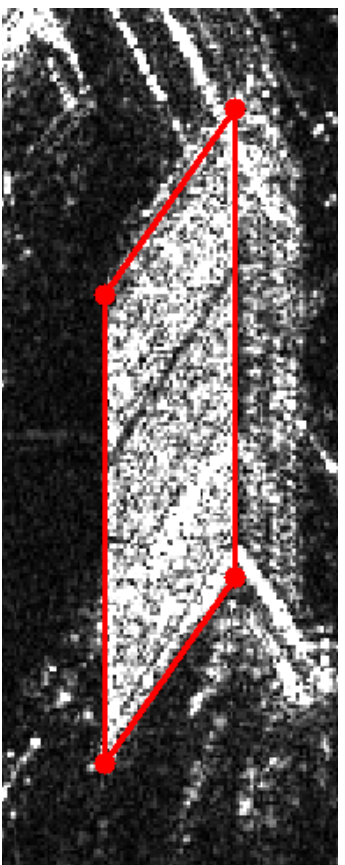

(c) $51^{\circ}, \mathrm{HH}$

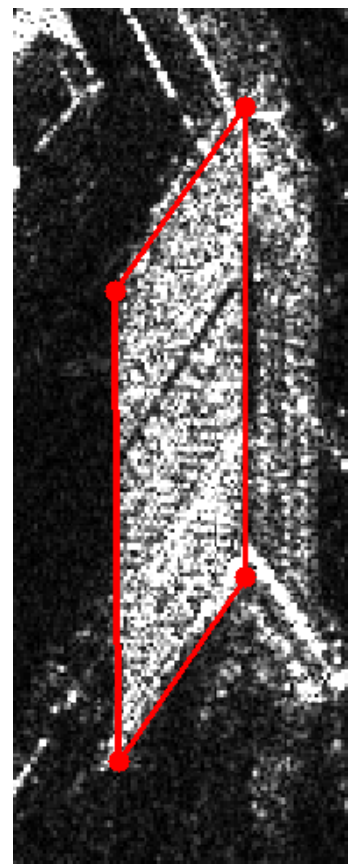

(d) $51^{\circ}, \mathrm{VV}$

Figure 4. Facade at Alexanderplatz, Berlin. Extracted facade layover areas indicated by red frames. Time difference between HH and VV data takes: 11 days.

\begin{tabular}{|l|l|l|l|l|l|l|l|}
\hline Building / data take & $\begin{array}{l}\text { Mean } \\
(\text { LO) }\end{array}$ & $\begin{array}{l}\text { Median } \\
\text { (LO) }\end{array}$ & $\begin{array}{l}\text { Std. Dev. } \\
\text { (LO) }\end{array}$ & $\begin{array}{l}\text { Mean } \\
(\text { Max })\end{array}$ & $\begin{array}{l}\text { Median } \\
(\text { Max })\end{array}$ & $\begin{array}{l}\text { Std. Dev. } \\
\text { (Max) }\end{array}$ & $\begin{array}{l}\text { No. } \\
\text { Max. }\end{array}$ \\
\hline \hline Hansaviertel, $\mathrm{HH}, 30^{\circ}$ & 4.06 & 4.07 & 1.01 & 4.87 & 4.81 & 0.79 & 409 \\
\hline Hansaviertel, VV, 30 ${ }^{\circ}$ & 3.67 & 3.69 & 0.95 & 4.48 & 4.32 & 0.72 & 359 \\
\hline Hansaviertel, $\mathrm{HH}, 51^{\circ}$ & 5.13 & 5.08 & 1.16 & 5.98 & 5.83 & 0.98 & 256 \\
\hline Hansaviertel, VV, 51 & 4.70 & 4.70 & 1.06 & 5.65 & 5.55 & 0.83 & 272 \\
\hline Alexanderplatz, $\mathrm{HH}, 30^{\circ}$ & 4.66 & 4.72 & 0.92 & 5.46 & 5.48 & 0.66 & 783 \\
\hline Alexanderplatz, $\mathrm{VV}, 30^{\circ}$ & 4.48 & 4.51 & 0.89 & 5.21 & 5.12 & 0.65 & 872 \\
\hline Alexanderplatz, $\mathrm{HH}, 51^{\circ}$ & 5.36 & 5.47 & 0.85 & 6.04 & 6.05 & 0.57 & 736 \\
\hline Alexanderplatz, $\mathrm{VV}, 51^{\circ}$ & 5.17 & 5.30 & 0.85 & 5.92 & 5.98 & 0.57 & 670 \\
\hline
\end{tabular}

Table 1. Summary of layover properties. LO = layover, Max = local maxima, No. Max. = number of maxima with intensity above mean layover intensity.

Cadastral and Surveying Authorities of the Länder of the Federal Republic of Germany provide CityGML building objects for the entire territory of Germany (Aringer and Roschlaub, 2013), the INSPIRE directive of the European Union allows EU member states to encode their data on buildings using the CityGML format (Groeger et al., 2013) and many cities around the World such as Doha, Kuala Lumpur, Yokohama and Zurich are applying CityGML for their 3D city models). Second, the CityGML data structure is adapted to the requirements of building reconstruction using data acquisition techniques (such as SAR) which allow to observe the boundary surfaces of real world objects (Nagel et al., 2009).

A realistic choice is made with respect to the level of detail in the context of SAR applications and with respect to CityGML data availability (LOD-1 and LOD-2). A higher level of detail may be preferable but hardly available. In this paper, LOD-2 is chosen for the simulation case study, roughly representing the geometry of roofs. Compared to geometric information from a DEM, several advantages are of interest in the context of SAR simulation under the assumption of a (geodetic) origin of the data and a thorough maintaining of the GIS data base:
- In contrast to a DSM, semantic objects are stored in a CityGML data set which allows for discriminating e.g. buildings from their surrounding vegetation.

- The coordinates of the building boundaries are defined as vector (boundary representation) data in 3D and presumably of high accuracy. In case of a DSM, 3D building boundaries have to be extracted from a 2.5D model where facades are not represented by vertical surfaces. As a consequence, the accuracy of the building outline is moderate.

- The building geometry (horizontal position and height) can be consecutively updated, e.g. by surveying campaigns. Updating a DSM requires much more effort, limiting the actuality of the data.

- The semantic classification of facade and roof parts is already given by the model. Methods for decomposing model parts (as required for the DSM; e.g. (Tao et al., 2014)) are not necessary.

- In addition to the geometry, valuable thematic information may be included in the data such as the current and the intended usage of the building, the year of construction and 


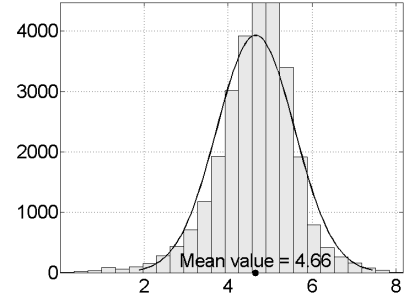

(a) Layover, $30^{\circ}, \mathrm{HH}$

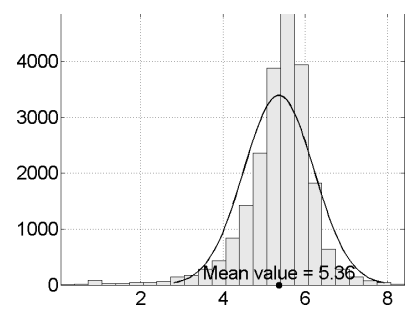

(c) Layover, $51^{\circ}, \mathrm{HH}$

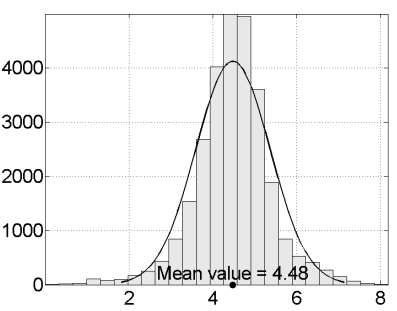

(b) Layover, $30^{\circ}, \mathrm{VV}$

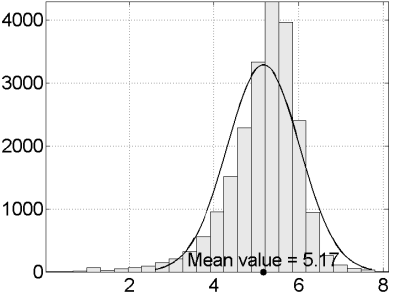

(d) Layover, $51^{\circ}, \mathrm{HH}$
Figure 5. Alexanderplatz, intensity distribution of layover pixels for different polarizations and incidence angles.

\begin{tabular}{|l|l|l|l|}
\hline Surface arrangement & $\theta_{i}$ & $\mathrm{H} \mathrm{Pol}$ & $\mathrm{V} \mathrm{Pol}$ \\
\hline \hline $\begin{array}{l}\text { Glas on first or second } \\
\text { surface }\end{array}$ & $15^{\circ}$ & 0.28 & 0.26 \\
\hline Glas on third surface & $15^{\circ}$ & 0.72 & 0.11 \\
\hline Arbitrary & $45^{\circ}$ & 0.38 & 0.17 \\
\hline $\begin{array}{l}\text { Glas on first or second } \\
\text { surface }\end{array}$ & $80^{\circ}$ & 0.82 & 0.21 \\
\hline Glas on third surface & $80^{\circ}$ & 0.27 & 0.26 \\
\hline
\end{tabular}

Table 2. Reflection component resulting at two metallic surfaces and one glass surface coated by 10 percent silver (no signal loss: 1) for different local signal incidence angles $\left(\theta_{i}\right)$ on first surface. Permittivity parameters assumed for $\mathbf{C}$ Band. Metal: $1.0+j * 3.4 * 10^{7}$, glass: 6.4, silver compound: $1.0+j * 2.1 * 10^{8}$. Note: High or low local signal incidence angles may occur for strong rotations of the corner with respect to the sensor's line-of-sight.

links to external information systems. Accordingly, SAR simulation case studies can be concentrated on specific model properties.

One major drawback is related to building walls/roofs if the geometry does not rely on measured data (example: height estimate based on height step multiplied with number of floors). Then, the correctness of the simulated building layover will be representative at the corner line (bottom line of facade; far-range) but only a rough approximate at the other end (near-range).

As a major objective, realizing an interface to CityGML increases the flexibility of the simulation processing chain which was concentrated on LiDAR data and DEMS so far (necessary to deal with urgent situations). As a next step, findings of the case study may be extended to other formats.

\subsection{Simulation based on CityGML}

The simulation based on CityGML requires a functional and fully automated interface between CityGML and GeoRaySAR. As a pre-requisite, the interface has to be developed in a way that it can be adapted to other GIS formats and other information models with low effort. Following this idea, the Feature Manipulation

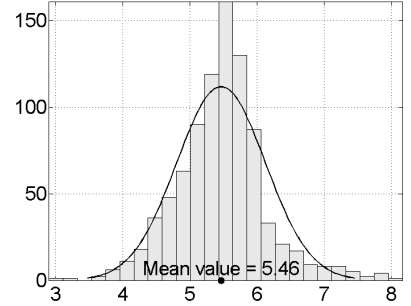

(a) Maxima, $30^{\circ}, \mathrm{HH}$

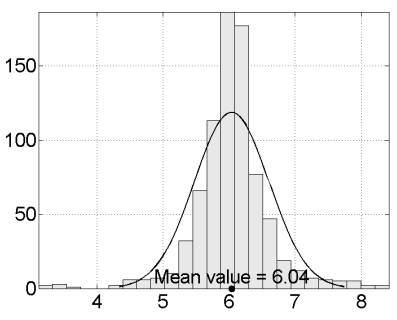

(c) Maxima, $51^{\circ}, \mathrm{HH}$

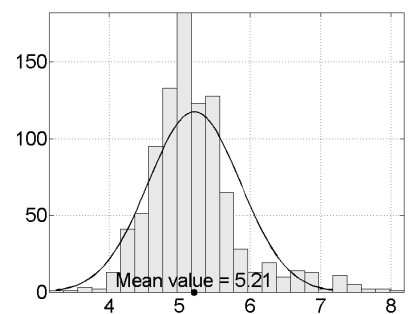

(b) Maxima, $30^{\circ}, \mathrm{VV}$

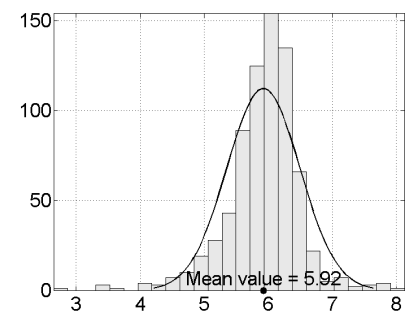

(d) Maxima, $51^{\circ}, \mathrm{VV}$
Figure 6. Alexanderplatz, intensity distribution of maxima for different polarizations and incidence angles.

Engine (FME) by Safe Software Inc. (http://www.safe.com) has been selected as an appropriate platform for own developments as it is flexible to a high number of different model formats. In more detail, imported model formats are parsed to a uniform model code in FME where model properties can be manipulated based on own strategies (tools: transformers). Note that the developed interface can be integrated into the simulation processing chain reported in (Tao et al., 2014).

As a first step, the urban scene of interest is extracted from the CityGML data set based on the corner coordinates of a defined frame. Thereafter, all surfaces of the model are defined as separate entities and grouped to building objects with own IDs. In this context, roof parts are represented by triangles (required: triangulation step) whereas walls are represented by flat polygons. If necessary, the model coordinates are transformed to the UTM coordinate system (example: CityGML data set defined in Gauss-Krueger (GK) coordinate system). Afterward, the polygons and triangles are decomposed to corner points, respectively, while counting the number of corners. For geocoding, the bounding box is needed for each scene object (see (Tao et al., 2014)). Accordingly, the bounding box is extracted for the full scene model as well as for each building model. For the sake of convenience, the origin of the POV-Ray coordinate system is always shifted to the gravity center of the building model (full scene or individual building), which therefore has to be calculated. No rotation is necessary due to absolute coordinates within in the CityGML data set. As a final step, the corner coordinates of roof and wall surfaces are written into strings which are used to generate a POV-Ray source file of the model based on polygons. To this end, the number of corner points is required for defining the objects. The resulting POV-Ray files define the scene geometry for SAR simulation (full scene or individual building). Together with the bounding box information, the scene definition is imported to the SAR simulation processing chain. Therein, the virtual antenna/sensor is added to the POV-Ray code based on the TerraSAR-X orbit file which enables to interpolate the local angle of incidence of the scene and to extract the sensor heading angle. Likewise, appropriate roughness is assigned to all surfaces in order to guarantee the prominent appearance of layover areas in the simulated SAR image. The surface roughness is used to represent direct backscattering but also triple reflections from spatially separated corner reflectors (quasi-direct response from corner tip), 


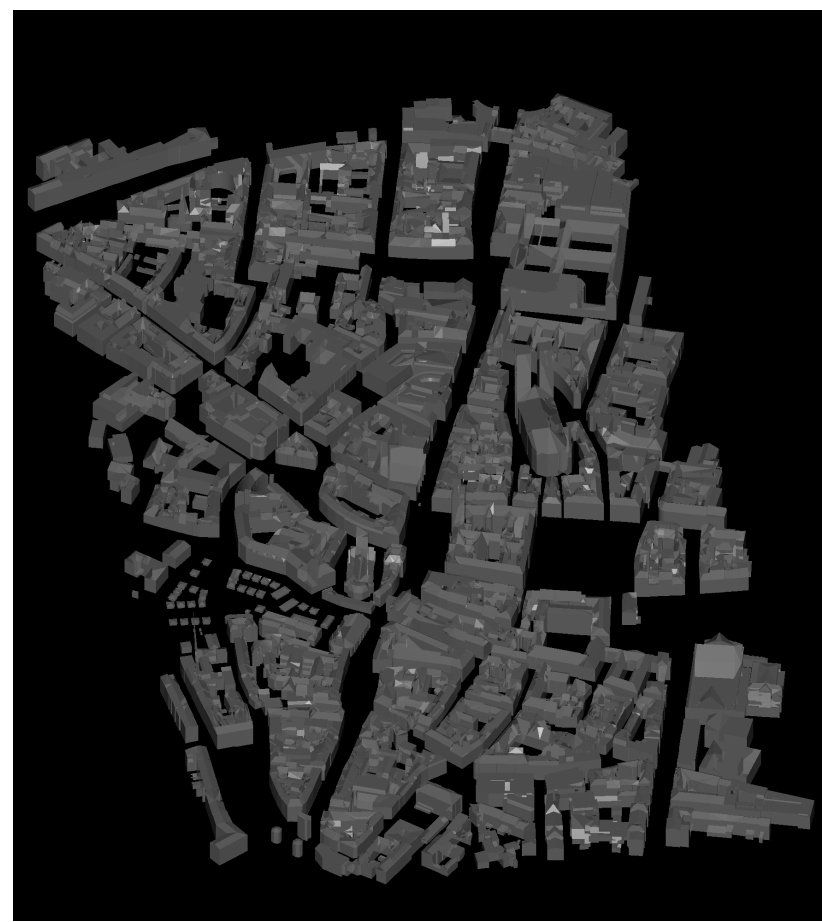

Figure 7. Rendered image of CityGML model, representing the line-of-sight of the virtual SAR sensor.

which are not represented by LOD-1 and LOD- 2 models. The reflection levels of the simulation are restricted to direct backscattering and double reflections as these can be directly related to buildings. Reflection levels beyond double reflection are deactivated as they are not representative due to missing details on the facade models (facades represented by plane polygons). The simulated SAR images are generated based on a ray tracing procedure (see details in (Auer, 2011)). Thereafter, the geocoding of the images is conducted using the bounding box information of the scene (Tao et al., 2014) (full scene or individual building). In that regard, differences in the height system are considered for avoiding a shift error in range direction. As an example, the difference between the ellipsoidal height and sea level height of the Munich city center is $45.52 \mathrm{~m}$ for the case study presented below.

\section{SIMULATION CASE STUDY}

As an appropriate test site, the Munich city center has been chosen where a high resolution TerraSAR-X data set and a CityGML model, derived from 2D building footprints from cadastral land register and photogrammetric measurements, are available. The geocoded TerraSAR-X image, captured in spotlight mode from a descending orbit with an incidence angle of $49.75^{\circ}$, has a pixel spacing of $0.5 \mathrm{~m} \times 0.5 \mathrm{~m}$. Being of type LOD-2, the CityGML data set includes a rough approximation of the roof geometry. The model coordinates, given in the GK coordinate system, have been transformed to the UTM coordinate system, which is realized when translating the geometry to the POV-Ray format. After adding the virtual sensor based on the TerraSAR-X orbit file to the POV-Ray model code, ray tracing is performed for detecting signal reflections on the model surface (diffuse, specular) and a SAR image is simulated whose pixel spacing is adapted to the TerraSAR-X image. As a bi-product, an optical image can be derived as well, indicating the sensor perspective with respect to the urban scene. Fig. 7 shows the rendered scene which is mainly characterized by residential buildings but also contains buildings of large (e.g. Frauenkirche on the center right) and small size (e.g. Viktualienmarkt on the center left). Foreshortening is distinguishable for some roofs which are hit perpendicular by the radar signal. Nonetheless, diffusely reflected signals are more important as they will represent the extent of building layover in the simulated SAR image, whereas signal contributions related to foreshortening and double reflections will be condensed to lines. Diffuse reflection from the ground is suppressed as the focus is on building layover. In contrast, specular reflections are assigned to ground parts in order to enable the simulation of double reflection lines. Accordingly, with except of the contribution to double reflection lines, ground parts are not distinguishable in the simulated SAR image.

Fig. 8(a) shows the resulting SAR image where building responses are represented by bright layover areas and partly double reflection lines. It is seen that the layover extent of large and small buildings shows prominent appearance which enables to define the corresponding layover extent in the azimuth-range plane. Geocoding performs a rotation of the image, considering the angle between the sensor heading direction and the north direction. For a visual inspection, the simulated image can be superposed to the TerraSAR-X image as shown in Fig. 8(b) (layover marked by red color).

Fig. 9 exemplifies the simulation of large and small urban structures by two examples within the urban scene. The layover corresponding to facade and roof structures include dominant signatures but also parts with little response. However, the simulated image enables to mark the related layover outline. Moreover, it is obvious that some building geometries are not represented by the CityGML model due to errors in data acquisition and modeling. This can also be seen in Fig. 8(b) where some newly constructed building blocks are missing in the simulation result (bright layover without red cover). For a distinct analysis of the buildings in the TerraSAR-X image, image parts related to building layover may be extracted based on an intensity threshold, following the concept reported in (Tao et al., 2014).

\section{POTENTIALS AND CHALLENGES OF CITYGML IN THE CONTEXT OF CHANGE DETECTION}

Simulations of the full scene enable to provide an overview of the scene in order to identify image parts related to building facades. Layover parts can be extracted in two SAR images in order to analyze changes of the pertinent intensity distributions or prominent features. Beyond this level, CityGML data provides additional opportunities compared to the use of digital surface models.

First, decomposing the scene model into smaller parts (building blocks or facades) as reported in (Tao et al., 2013) is not necessary as building components are already defined in the CityGML data sets. Hence, model errors (loss of buildings or building parts due to height steps and small dimension; unintended splitting) and inaccuracies (building outline; non-vertical walls) related to the model decomposition are avoided. The analysis is further extendable to individual facades (not shown in this paper). Second, semantic information can be considered when analyzing changes. For instance, the focus can be set on buildings with high priority in case of urgent situations. Buildings can be filtered by the usage and data from external information systems referenced by the CityGML object. Accordingly, the focus can be set, e.g., on residential buildings, schools, hospitals, train stations or production facilities which handle hazardous goods in urgent situations in order to support local rescue teams. Moreover, densely populated areas or critical infrastructure (bridges, major roads, and railway links) can be detected from the CityGML data set for prioritizing change detection. Third, CityGML data enable to consider temporal aspects. The CityGML data set can be updated 


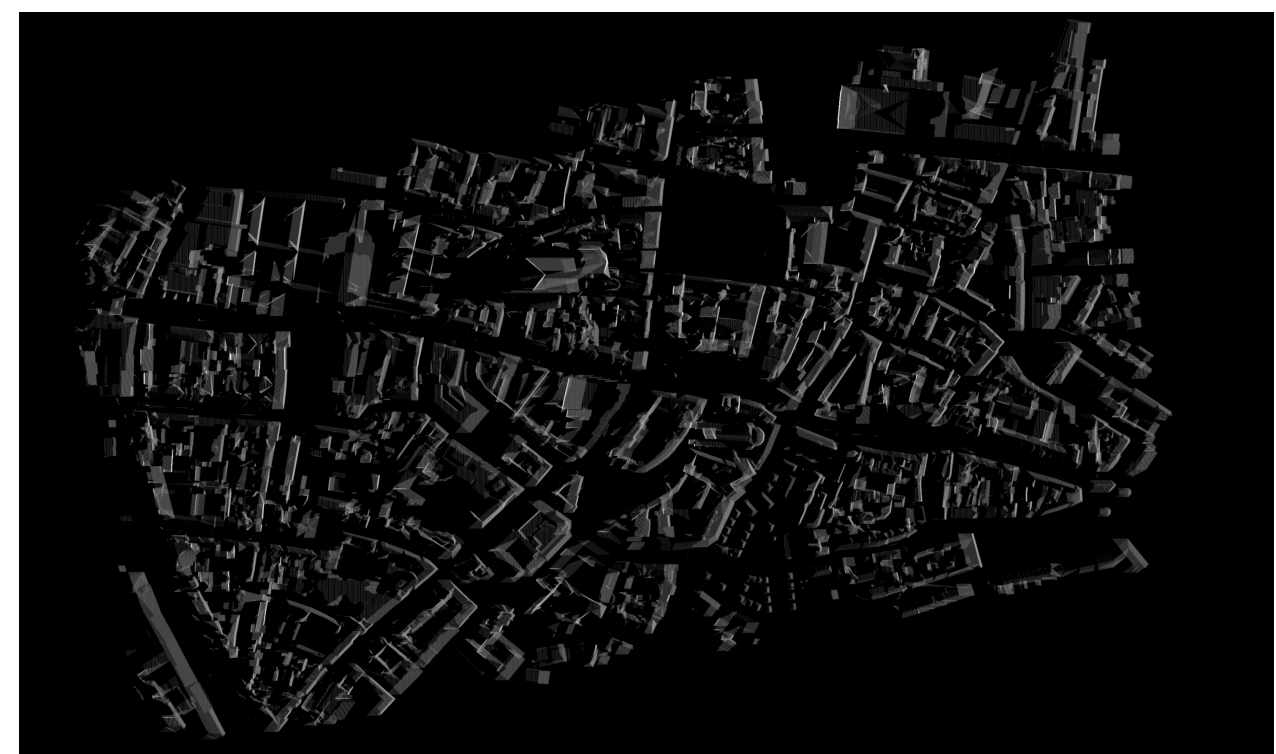

(a) Simulated reflectivity map with pixel spacing adapted to TerraSAR-X image $(0.5 \mathrm{~m} \mathrm{x} 0.5 \mathrm{~m})$. Included reflection levels: direct backscattering and double reflection.

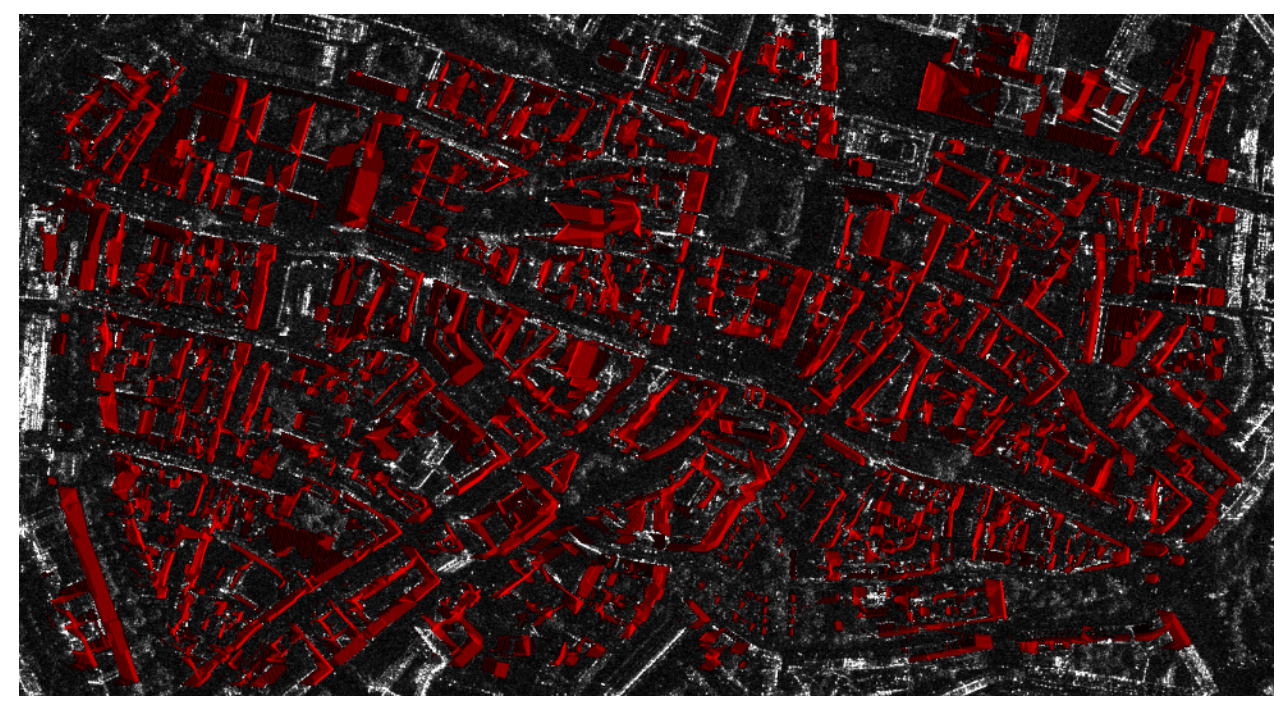

(b) Reflectivity map superposed on geocoded spotlight TerraSAR-X image.

Figure 8. Simulation result based on CityGML model.

continuously with limited effort (based on dedicated field surveys) whereas updates of DSMs commonly depend on new flight campaigns based on optical sensors or laser scanning. Moreover, individual city objects can be filtered by the transaction time of the data (CityGML attributes "creationDate" and "terminationDate"). Thereby, the varying actuality of the geometric and semantic information can be considered. Finally, buildings can be filtered by considering the valid time (CityGML attributes "yearOfConstruction" and "yearOfDemolition") in order to adapt the comparability to the SAR data. As a drawback, though, the accuracy of building heights in the CityGML may be moderate, e.g., due to height estimates.

As CityGML not only allows for representing objects in several levels of detail but also for geometrically representing objects in a number of ways (e.g. an LOD2-Building may be represented by a single Solid geometry as well as by several MultiSurface geometries and its outer shell may or may not be decomposed in semantic boundary surface objects), the transformation process has to account for all these variations and can therefore become rather complex. With regard to the thematic information contained in a CityGML data set, on the one hand the transformation process should be able to exploit all the thematic information, e.g. for filtering the data as described above. As most of the thematic attributes of the CityGML information model are optional, the transformation process on the other hand, should be able to provide valuable input to the SAR simulation even if the thematic information is not available.

\section{CONCLUSION AND OUTLOOK}

The work presented in this paper addresses two aspects: A.) the appearance of facade layover in the context of different signal incidence angles and signal polarizations and B.) the identification of building-related image parts in high resolution SAR images. The close-up view on TerraSAR-X images indicates that the visual perception of layover varies significantly while the intensity distributions intend to follow log-normal distributions (even for local maxima as a rough approximate). The polarization mode 

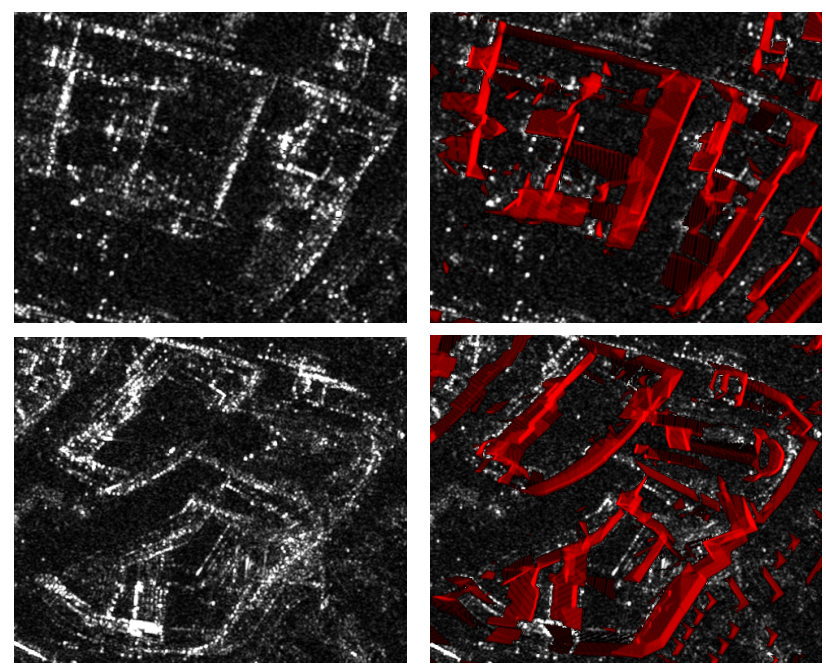

Figure 9. Close-up view on building blocks.

HH dominates VV in intensity (mean, median) but also tends to show stronger intensity variation (standard deviation). In contrast, no tendency with respect to the proportion between $\mathrm{HH}$ and VV is observed on the number of prominent point signatures. As a conclusion, distribution-based methods for layover analysis seem to be favored which may be optionally supported by featurebased concepts, e.g., focused on lines or point-like signatures.

The prominent appearance of facade layover motivates the identification of the related SAR image parts in order to extract buildingrelated information. In this context, a concept has been proposed for identifying building layover based on simulation methods including CityGML data. To this end, a simulation processing chain has been extended to fuse TerraSAR-X images and prior information provided by CityGML data sets. In this context, the data transformation from CityGML to the POV-Ray data structure used by the simulator is fully automated using the spatial ETL (extract, transform, and load) software FME. As a first example, a case study of the Munich city center has been shown where the extent of simulated building layover is directly superposed on a geocoded TerraSAR-X image. The simulation concept based on CityGML data indicates that the representation of real world entities by semantic objects has a number of advantages over the purely geometric representation in a DSM. In particular, change detection applications may benefit from CityGML data sets if they are kept up to date and assigned with meta information.

Future work will be related to enhancements of the interface between simulation and GIS data. Different levels of detail (LODs) and model formats will have to be tested for their use and interfaces. The simulation results of the case studies are related to the full scene model. As a next step, the analysis will be continued on the level of individual buildings and facades.

\section{ACKNOWLEDGEMENTS}

The authors want to thank Isabel Hornig and Martin Pültz for their support in the layover analysis and CityGML integration, respectively. The work was partly funded by the Deutsche Forschungsgemeinschaft (DFG), project VHR-SAR (BA 2033/3-1).

\section{REFERENCES}

Aringer, K. and Roschlaub, R., 2013. Calculation and update of a 3D building model of bacaria using LiDAR, image matching and catastre information. In: Int. Arch. Photogramm. Remote Sens., Vol. II-2/W1.

Auer, S., 2011. 3D Synthetic Aperture Radar Simulation for Interpreting Complex Urban Reflection Scenarios. PhD thesis, Deutsche Geodätische Kommission, Reihe C, Nr. 660, Verlag der Bayerischen Akademie der Wissenschaften, $126 \mathrm{p}$.

Auer, S., Gisinger, C. and Tao, J., 2015. Characterization of facade regularities in high-resolution SAR images. IEEE Trans. Geosci. Remote Sens. 53(5), pp. 2727-2737.

Ferro, A., Brunner, D. and Bruzzone, L., 2013. Automatic detection and reconstruction of building radar footprints from single VHR SAR images. IEEE Trans. Geosci. Remote Sens. 51(2), pp. $935-952$.

Gernhardt, S. and Bamler, R., 2012. Deformation monitoring of single buildings using meter-resolution SAR data in PSI. ISPRS J. Photogramm. Remote Sens. 73, pp. 68-79.

Groeger, G., Kolbe, T. H., Nagel, C. and Haefele, K.-H., 2012. OGC City Geography Markup Language (CityGML) Encoding Standard, v2.0. OGC Doc. No. 12-019. Open Geospatial Consortium.

Groeger, G., Kutzner, T. and Kolbe, T. H., 2013. A CityGMLbased encoding for the INSPIRE data specification on buildings. In: INSPIRE 2013: The Green Renaissance.

Michaelsen, E., Soergel, U. and Thoennessen, U., 2006. Perceptual grouping for automatic detection of man-made structures in high-resolution SAR data. Pattern Recogn. Lett. 27(4), pp. 218225 .

Nagel, C., Stadler, A. and Kolbe, T. H., 2009. Conceptual requirements for the automatic reconstruction of building information models from uninterpreted 3D models. In: Int. Arch. Photogramm. Remote Sens., Vol. Vol. 34, Part XXX.

Oliver, C. and Quegan, S., 2004. Understanding Synthetic Aperture Images. SciTech Publishing, Inc.

Simonetto, E., Oriot, H. and Garello, R., 2005. Rectangular building extraction from stereoscopic airborne radar images. IEEE Trans. Geosci. Remote Sens. 43(10), pp. 2386-2395.

Soergel, U., Thoennessen, U., Brenner, A. and Stilla, U., 2006. High-resolution SAR data: new opportunities and challenges for the analysis of urban areas. IEE Proceedings - Radar, Sonar and Navigation 153(3), pp. 294-300.

Tao, J., Auer, S., Palubinskas, G., Reinartz, P. and Bamler, R., 2014. Automatic SAR simulation technique for object identification in complex urban scenarios. IEEE J. Sel. Topics Appl. Earth Observ. 7(3), pp. 994-1003.

Tao, J., Auer, S., Reinartz, P. and Bamler, R., 2013. Object-based change detection for individual buildings in SAR images captured with different incidence angles. In: Proceedings of IGARSS.

Thiele, A., Wurth, M., Even, M. and Hinz, S., 2013. Extraction of building shape from TanDEM-X data. In: Int. Arch. Photogramm. Remote Sens., Vol. XL-1/W1.

Wegner, J., Auer, S. and Soergel, U., 2010. Geometrical investigation of SAR double-bounce lines. Photogramm. Eng. Remote Sens. 76(9), pp. 1071-1080. 